\title{
Integrable deformations of local analytic fibrations with singularities
}

\author{
Dominique Cerveau and Bruno Scárdua
}

\begin{abstract}
We study analytic integrable deformations of the germ of a holomorphic foliation given by $d f=0$ at the origin $0 \in \mathbb{C}^{n}, n \geq 3$. We consider the case where $f$ is a germ of an irreducible and reduced holomorphic function. Our central hypotheses is that, outside of a dimension $\leq n-3$ analytic subset $Y \subset X$, the analytic hypersurface $X_{f}:(f=0)$ has only normal crossings singularities. We then prove that, as germs, such deformations also exhibit a holomorphic first integral, depending analytically on the parameter of the deformation. This applies to the study of integrable germs writing as $\omega=d f+f \eta$ where $f$ is quasi-homogeneous. Under the same hypotheses for $X_{f}:(f=0)$ we prove that $\omega$ also admits a holomorphic first integral. Finally, we conclude that an integrable germ $\omega=a d f+f \eta$ admits a holomorphic first integral provided that: (i) $X_{f}:(f=0)$ is irreducible with an isolated singularity at the origin $0 \in \mathbb{C}^{n}, n \geq 3$; (ii) the algebraic multiplicities of $\omega$ and $f$ at the origin satisfy $\nu(\omega)=\nu(d f)$. In the case of an isolated singularity for $(f=0)$ the writing $\omega=a d f+f \eta$ is always assured so that we conclude the existence of a holomorphic first integral. Some questions related to Relative Cohomology are naturally considered and not all of them answered.
\end{abstract}

\section{Introduction and main results}

The problem of integrability of differential equations in the real context goes back to H. Poincaré and Dulac. In the analytic case it is natural to consider the complexification of the equation and then we are just one step away from the holomorphic foliations framework. These are objects that can be described by integrable systems of (holomorphic) one-forms. Under this viewpoint probably the most important result is Malgrange's work ([12] and [13]), relating the dimension of the singular set of the system with the existence of a holomorphic first integral for it. This is one of the motivations for this work. 


\subsection{Main results}

We consider $f: \mathbb{C}^{n}, 0 \rightarrow \mathbb{C}, 0$ a germ of a holomorphic function at the origin $0 \in \mathbb{C}^{n}, n \geq 3$. The corresponding germ of an analytic hypersurface $(f=0)$ is denoted by $X_{f}$. The singular set of the hypersurface $X_{f}$ will be denoted by $\operatorname{sing}\left(X_{f}\right)$. We will denote by $X_{f}^{*}=X_{f}-\operatorname{sing}\left(X_{f}\right)$ the smooth part of $X$. Next, we give a pleonastic definition of our main hypothesis:

Definition 1.1. We shall say that $X_{f}$ has only ordinary singularities off a codimension $\geq 3$ subset if there exists an analytic subset $(Y, 0) \subset\left(X_{f}, 0\right)$ of dimension at most $n-3$, such that outside of $Y$ the only singularities of $\left(X_{f}, 0\right)$ are normal crossings.

We will assume that $f$ is reduced (if $g \in \mathcal{O}_{n}$ is such that $\left.g\right|_{X_{f}} \equiv 0$ then $f \mid g$ in $\mathcal{O}_{n}$ ). In this case the singular set of $X_{f}$ is given by $\operatorname{sing}\left(X_{f}\right)=\operatorname{sing}(f)=\left\{p \in\left(\mathbb{C}^{n}, 0\right): d f(p)=\right.$ $0\}$. Indeed, it is well-known ([15]) that the singular points of $f$, i.e., the zeroes of $d f$, are contained in the fiber $f^{-1}(0)$. We consider the germ of an integrable one-form $\omega \in \Omega^{1}\left(\mathbb{C}^{n}, 0\right)$. Then $\omega=0$ defines a codimension-one holomorphic foliation $\mathcal{F}(\omega)$ germ at $0 \in \mathbb{C}^{n}$. The hypersurface $X_{f}$ is $\mathcal{F}(\omega)$-invariant if, and only if, $\omega \wedge d f / f$ is holomorphic ([1] and [16]). This is the case of integrable one-forms that write as

$$
\omega=a d f+f \eta
$$

with $a \in \mathcal{O}_{n}$ and $\eta \in \Omega^{1}\left(\mathbb{C}^{n}, 0\right)$.

In a certain sense this is the most natural writing for a one-form $\omega$ that leaves $X_{f}$ invariant (see Section 4 and Section 5).

For $\eta$ small enough (in the sense of Krull topology [8] and [9]) and $a \in \mathcal{O}_{n}^{*}$ unit, we may see $\mathcal{F}(\omega)$ as an integrable deformation of the holomorphic "fibration" $\mathcal{F}(d f)$, given by $f=$ const. If, for instance, $f$ has an isolated singularity at $0 \in \mathbb{C}^{n}$, $n \geq 3$, then any $\omega$ that leaves $X_{f}:(f=0)$ invariant must write as above, $\omega=a d f+f \eta$. In particular, $\omega$ may come from an analytic deformation of $\omega_{0}=d f$, under some geometrical condition (e.g., if $\nu(\omega)=\nu(d f)$ as explained in the text itself).

We will consider the following situation: $\left\{\omega_{t}, t \in(\mathbb{C}, 0)\right\}$ is an analytic deformation of $\omega_{0}=d f$ such that each one-form $\omega_{t} \in \Omega^{1}\left(\mathbb{C}^{n}, 0\right)$ is integrable, $\omega_{t} \wedge d \omega_{t}=0$.

We then prove in Section 2:

Theorem 1.2. Assume that the germ $f \in \mathcal{O}_{n}, n \geq 3$ is reduced, $X_{f}$ is irreducible and has only normal crossings singularities off a codimension $\geq 3$ subset. Let $\left\{\omega_{t}\right\}_{t \in \mathbb{C}, 0}$ be an analytic deformation of $\omega_{0}=d f$ at $0 \in \mathbb{C}^{n}$. Then for any $t \in(\mathbb{C}, 0)$ close enough to 0 , the one-form $\omega_{t}$ admits a holomorphic first integral. Indeed, there is a germ of a holomorphic function $F:\left(\mathbb{C}^{n} \times \mathbb{C}, 0\right) \rightarrow(\mathbb{C}, 0),(x, t) \mapsto F(x, t)$ such that: 
(1) $F(x, 0)=f(x)$

(2) $F_{t}: x \mapsto F(x, t)$ is a first integral for $\omega_{t}$.

Remark 1.3. Regarding the hypotheses in Theorem 1.2, we observe that:

(1) It is enough to assume that they hold for the restriction of $\omega$ to a threedimensional plane, in general position with respect to $\omega$, in the same sense of [14].

Indeed, according to [14] if such a restriction admits a holomorphic first integral, then the same holds for the form $\omega$. Actually, it is proved that the first integral for the 3-dimensional plane section admits an extension to a first integral for the form $\omega$ in a neighborhood of the origin $0 \in \mathbb{C}^{n}$.

(2) In particular, if there is a three-dimension plane section of $X$ which has an isolated singularity at the origin, then the conclusion of Theorem 1.2 is valid.

As a corollary we have (see Section 4):

Theorem 1.4. Let $f \in \mathcal{O}_{n}, n \geq 3$ be a strictly quasi-homogeneous reduced function. Assume that $X_{f}:(f=0)$ is irreducible and has only normal crossings singularities off a codimension $\geq 3$ subset. Then, any holomorphic integrable one-form germ $\omega \in \Omega^{1}\left(\mathbb{C}^{n}, 0\right)$ of the form $\omega=d f+f \eta$, with $\eta \in \Omega^{1}\left(\mathbb{C}^{n}, 0\right)$, admits a holomorphic first integral.

Remark 1.5. A function $f\left(x_{1}, \ldots, x_{n}\right)$ is quasi-homogeneous if there exist $d, d_{1}$, $\ldots, d_{n}$ all non-negative such that $f\left(t^{d_{1}} x_{1}, \ldots, t^{d_{n}} x_{n}\right)=t^{d} f\left(x_{1}, \ldots, x_{n}\right)$. It is strictly quasi-homogeneous if $d>0$ and $d_{j}>0, \forall j$.

Then, as a consequence of our approach and some relative cohomology results based on [1] and [16] we obtain, for the case of an isolated singularity (cf. Section 4):

Theorem 1.6. Let $f \in \mathcal{O}_{n}$ be reduced and irreducible, with an isolated singularity at the origin. Let $\omega \in \Omega^{1}\left(\mathbb{C}^{n}, 0\right), n \geq 3$ be an integrable one-form having $(f=0)$ as the only invariant hypersurface. Then $\omega$ admits a (germ of a) holomorphic first integral if, and only if, $\nu(\omega)=\nu(d f)$ at 0 .

In the above statement, $\nu($.$) stands for the algebraic multiplicity at the origin.$

\section{Germs of hypersurfaces with normal crossings}

We consider $\left(X_{f}, 0\right) \subset\left(\mathbb{C}^{n}, 0\right)$ a germ of reduced analytic surface in $\left(\mathbb{C}^{n}, 0\right)$, defined by $f=0$ where $f \in \mathcal{O}_{\mathbb{C}^{n}, 0}$ is a germ of analytic function in $0 \in \mathbb{C}^{n}$. If $n=3$ and $\left(X_{f}, 0\right)$ only has normal crossings singularities off the origin $0 \in \mathbb{C}^{3}$, then the local fundamental group of the complement of $\left(X_{f}, 0\right)$ in $\left(\mathbb{C}^{n}, 0\right)$ is abelian. This is a particular case of the more general statement below: 
Theorem 2.1. (Lê-Saito, [10] Main Theorem page 1) Let $n \geq 3$. Assume that outside of an analytic subset $(Y, 0) \subset\left(X_{f}, 0\right)$ of dimension at most $n-3$, the only singularities of $\left(X_{f}, 0\right)$ are normal crossings. Then the local fundamental group of the complement of $\left(X_{f}, 0\right)$ in $\left(\mathbb{C}^{n}, 0\right)$ is abelian. The Milnor fiber of $f$ has a fundamental group which is free abelian of rank the number of analytic components of $X_{f}$ at 0 , minus one. Finally, if $X_{f}$ is irreducible, then the fiber $f^{-1}(c), c \neq 0$ is simply-connected.

Throughout this section, we will consider the case where $X=X_{f_{0}}$ is irreducible, given by $f_{0}=0$ as above with normal crossings singularities off a codimension $\geq 3$ subset. We study analytic integrable deformations of the one-form $\omega_{0}=d f_{0}$. Such a deformation writes as

$$
\omega_{t}=\omega_{0}+t \omega_{1}+\ldots+t^{k} \omega_{k}+\ldots
$$

where $t \in \mathbb{C}, 0$ and the $\omega_{j}$ are holomorphic in some small neighborhood $U$ of $0 \in$ $\mathbb{C}^{n}, n \geq 3, \forall j \geq 0$. The integrability condition $\omega_{t} \wedge d \omega_{t}=0$ gives:

$$
\begin{gathered}
\omega_{0} \wedge d \omega_{0}=0 \\
\omega_{0} \wedge d \omega_{1}+\omega_{1} \wedge d \omega_{0}=0 \\
\omega_{2} \wedge d \omega_{0}+\omega_{1} \wedge d \omega_{1}+\omega_{0} \wedge d \omega_{2}=0
\end{gathered}
$$

In our case $\omega_{0}=d f_{0}$, i.e., we have $d \omega_{0}=0$ and then

$$
d f_{0} \wedge d \omega_{1}=0
$$

and

$$
\omega_{1} \wedge d \omega_{1}+d f_{0} \wedge d \omega_{2}=0
$$

Now, $\omega_{1}$ is not necessarily integrable, but we have the following Relative Cohomology lemma:

Lemma 2.2. Under the above hypotheses we have:

$$
d \omega_{1} \wedge d f_{0}=0 \Longrightarrow \omega_{1}=d f_{1}+a_{1} d f_{0}
$$

for some holomorphic functions $f_{1}, a_{1}$ in $U$.

The proof of Lemma 2.2 is given in Section 5 as a consequence of Theorem 2.1 and some relative cohomology techniques adapted from [1].

Remark 2.3. The writing $\omega_{1}=d f_{1}+a_{1} d f_{0}$ is not unique but if $\omega_{1}=d \tilde{f}_{1}+\tilde{a}_{1} d f_{0}$ then $\tilde{f}_{1}=f_{1}+\varphi\left(f_{0}\right)$ and $a_{1}=\tilde{a}_{1}+\varphi^{\prime}\left(f_{0}\right)$ for some one-variable holomorphic germ $\varphi(z)$. 
Returning to the deformations we obtain $\omega_{t}=\omega_{0}+t \omega_{1}+t^{2} \omega_{2}+\ldots=d f_{0}+t\left(d f_{1}+a_{1} d f_{0}\right)+t^{2} \omega_{2}+\ldots=\left(1+t a_{1}\right) d f_{0}+t d f_{1}+t^{2} \omega_{2}+\ldots$

Therefore

$$
\frac{1}{1+t a_{1}} \omega_{t}=d f_{0}+\frac{t}{1+t a_{1}} d f_{1}+\frac{t^{2}}{1+t a_{1}} \omega_{2}+\ldots=d\left(f_{0}+t f_{1}\right)+t^{2} \widetilde{\omega}_{2}+\ldots
$$

We put

$$
\widetilde{\omega}_{t}=\frac{1}{1+t a_{1}} \omega_{t}
$$

recalling that $1+t a_{1}$ is a unit and $\widetilde{\omega}_{t}$ is also integrable holomorphic. Then we write

$$
\begin{gathered}
\widetilde{\omega}_{t}=d f_{0}+t d f_{1}+t^{2} \widetilde{\omega}_{2}+\widetilde{\omega}_{3}+\ldots \\
d \widetilde{\omega}_{t}=t^{2} d \widetilde{\omega}_{2}+t^{3} d \widetilde{\omega}_{3}+\ldots
\end{gathered}
$$

From the integrability condition we then obtain $d f_{0} \wedge d \widetilde{\omega}_{2}=0$. As above we obtain

$$
\widetilde{\omega}_{2}=d f_{2}+a_{2} d f_{0}
$$

for some holomorphic $a_{2}, f_{2}: U \rightarrow \mathbb{C}$. Then

$$
\widetilde{\omega}_{t}=d f_{0}+t d f_{1}+t^{2}\left(d f_{2}+a_{2} d f_{0}\right)+t^{3} \widetilde{\omega}_{3}+\ldots=\left(1+t^{2} a_{2}\right) d f_{0}+t d f_{1}+t^{2} d f_{2}+t^{3} \widetilde{\omega}_{3}+\ldots
$$

Hence, as above, we can define holomorphic integrable

$$
\widetilde{\tilde{\omega}_{t}}:=\frac{1}{1+t^{2} a_{2}} \widetilde{\omega}_{t}=d f_{0}+t d f_{1}+t^{2} d f_{2} t^{3} \widetilde{\widetilde{\omega}}_{3}+\ldots
$$

Inductively proceeding like this we obtain a formal unit $\widehat{G}$ such that

$$
\frac{1}{\widehat{G}} \omega_{t}=d f_{0}+\sum_{j=1}^{\infty} t^{j} d f_{j}
$$

for some holomorphic functions $f_{j}: U \rightarrow \mathbb{C}, j \geq 1$.

Thus we can write $\omega_{t}=\widehat{G}(x, t) \cdot d_{x} \widehat{F}(x, t)$ in the obvious sense for formal function $\widehat{F}(x, t)$ (indeed, $\widehat{F}$ is what is referred to as "transversely formal" in the sense of Mattei-Moussu).

Remark that there is a formal series $\hat{h}(x, t)$ such that $\omega_{t}+\hat{h} d t=\widehat{G} d_{(x, t)} \widehat{F}$.

Now we consider the pair $\{\Omega, d t\}$ where $\Omega(x, t)=\omega_{t}(x)$, defined in $(U \times \mathbb{C}, 0) \subset$ $\left(\mathbb{C}^{n+1}, 0\right)$. We claim that this is an integrable system: Indeed, $d_{(x, t)} \Omega=d_{x} \omega_{t}+\frac{\partial \omega_{t}}{\partial t} d t$ so that

$$
\Omega \wedge d \Omega \wedge d t=\omega_{t} \wedge d_{x} \omega_{t} \wedge d t+\omega_{t} \wedge \frac{\partial \omega_{t}}{\partial t} d t \wedge d t=0
$$

because we have $\omega_{t} \wedge d_{x} \omega_{t}=0$. 
We also claim that $\{\omega, d t\}=\{d \widehat{F}, d t\}$ at the level of formal modules. This is immediate from the expression $\Omega=\widehat{G} d \widehat{F}-\hat{h} d t$ and from the fact that $\widehat{G}$ is a unit. Now we recall the following result of Malgrange:

Theorem 2.4. (Malgrange, [13]) Let be given $p$ germs of holomorphic oneforms $\omega_{1}, \ldots, \omega_{p} \in \Omega^{1}\left(\mathbb{C}^{n}, 0\right)$ at the origin $0 \in \mathbb{C}^{n}$ with $1 \leq p \leq n$. Denote by $\mathcal{S}$ the germ of analytic set given by the zeros of $\omega_{1} \wedge \ldots \wedge \omega_{p}$. Assume that one of the conditions below is verified:

(i) $\operatorname{cod} \mathcal{S} \geq 3$ and $d \omega_{j} \wedge \omega_{1} \ldots \wedge \omega_{p}=0, \forall j$.

(ii) $\operatorname{cod} S \geq 2$ and $\left\{\omega_{1}, \ldots, \omega_{p}\right\}$ is formally integrable (i.e., there exist $\hat{f}_{i}, \hat{g}_{i j} \in \widehat{\mathcal{O}}_{n}$ such that $\omega_{i}=\sum_{j} \hat{g}_{i j} d \hat{f}_{j}, \forall i$ and $\left.\operatorname{det}\left(\hat{g}_{i j}\right)(0) \neq 0\right)$.

Then $\left\{\omega_{1}, \ldots, \omega_{p}\right\}$ is integrable meaning that there exist $f_{i}, g_{i j} \in \mathcal{O}_{n}$ (convergent) such that $\omega_{i}=\sum_{j} g_{i j} d f_{j}, \forall i$ and $\operatorname{det}\left(g_{i j}(0)\right) \neq 0$.

We shall apply this result to the system $\{\Omega, d t\}$. Recall that $\widehat{\mathcal{O}}\{\Omega, d t\}=$ $\{d \widehat{F}, d t\}$, that is, $\{\Omega, d t\}$ is formally integrable. Notice that $\Omega \wedge d t=d_{x} f_{0} \wedge d t+$ $\sum_{j \geq 1} t^{j} \omega_{j} \wedge d t$. Since cod $\operatorname{sing}\left(d f_{0}\right) \geq 2$ we conclude that $\operatorname{cod} \operatorname{sing}(\Omega \wedge d t) \geq 2$.

Thus, by (ii) in Malgrange's theorem above we have that $\{d \widehat{F}, d t\}$ admits a holomorphic (convergent) pair $\{d F, d t\}$. i.e., there is a holomorphic function $F(x, t)$ in a neighborhood of $0 \in \mathbb{C}^{n}$ such that $\Omega=a . d_{x} F(\cdot, t)+b d t$ for some holomorphic functions $a, b$ where $a$ is a unit. Therefore $\omega_{t}=a \cdot d_{x} F(x, t)$. This completes the proof of Theorem 1.2.

\section{Necessity of hypothesis, proof of Theorem 1.4}

We shall now discuss the necessity of our central hypothesis, about the normal crossings for the singularities of the hypersurface $X:(f=0)$.

Example 3.1. We consider $f_{0}=x^{3}+y^{2}$ and the corresponding cusp $\left(f_{0}=0\right)$ in $\left(\mathbb{C}^{2}, 0\right)$.

The cylinder generated by this cusp in $\left(\mathbb{C}^{3}, 0\right)$ is a hypersurface $X$ with singular set irreducible of codimension two. We consider deformations of the form

$$
\omega_{t}=d\left(y^{2}+x^{3}\right)+t x(2 x d y-3 y d x)
$$

From [3] and [11] it is known that there are no holomorphic first integrals for such a generic deformation. Embedding this on $\mathbb{C}^{3}, 0$ we conclude that the hypothesis of normal crossings for the hypersurface singularities cannot be dropped.

Proof of Theorem 1.4. We prove Theorem 1.4 as an application of Theorem 1.2 for the case of quasi-homogeneous hypersurface $X_{f}$. We start with a holomorphic 
integrable germ of one-form at $0 \in \mathbb{C}^{n}, n \geq 3$ of the following type

$$
\omega=d f+f \eta
$$

where $f$ is a strictly quasi-homogeneous function with normal crossings. This means that:

(1) there exist $d, d_{1}, \ldots, d_{n}>0$, such that $f\left(t^{d_{1}} x_{1}, \ldots, t^{d_{n}} x_{n}\right)=t^{d} f\left(x_{1}, \ldots, x_{n}\right)$;

(2) $X_{f}$ is an analytic irreducible hypersurface with ordinary (normal) crossings singularities off a codimension $\geq 3$ analytic subset.

Let us see how to consider (embed) $\omega$ as (into) a deformation of $\omega_{0}=d f$.

Take the map $\sigma_{t}: \mathbb{C}^{n}, 0 \rightarrow \mathbb{C}^{n}, 0$ defined for $t \neq 0$ by $\sigma_{t}(x)=\left(t^{d_{1}} x_{1}, \ldots, t^{d_{n}} x_{n}\right)$. Then we have $\sigma_{t}^{*} f=f \circ \sigma_{t}=t^{d} f$ and

$$
\sigma_{t}^{*} \omega=\sigma_{t}^{*}(d f+f \eta)=t^{d} d f+t^{d} f \sigma_{t}^{*}(\eta) .
$$

We then define $\omega_{t}:=\frac{1}{t^{d}} \sigma_{t}^{*} \omega=d f+f \sigma_{t}^{*}(\eta)$.

Notice that, because $d_{j}>0, \forall j$ we have $\sigma_{t}^{*}(\eta)=t \tilde{\eta}$ for some holomorphic oneform $\tilde{\eta}(x, t)$. Therefore $\omega_{t}=\frac{1}{t^{d}} \sigma_{t}^{*} \omega=d f+t f \tilde{\eta}$. Thanks to this form, $\omega_{t}$ is an analytic deformation of $\omega_{0}=d f$, such that $\omega_{1}=\omega$. Theorem 1.4 then follows from the fact that $\sigma_{t}$ defines an analytic diffeomorphism taking $\mathcal{F}\left(\omega_{1}\right)$ onto $\mathcal{F}\left(\omega_{t}\right)$ for all $t \neq 0$, and that from Theorem 1.2 the foliation $\mathcal{F}\left(\omega_{t}\right)$ admits a holomorphic first integral for $t$ close enough to 0 .

\section{The case of an isolated singularity}

We consider an integrable germ of holomorphic one-form $\omega \in \Omega^{1}\left(\mathbb{C}^{n}, 0\right)$ with an invariant hypersurface $X_{f}:(f=0)$ such that

(Is.1) $\operatorname{cod} \operatorname{sing}(\omega) \geq 2$

(Is.2) $X_{f}$ has an isolated singularity at $0 \in \mathbb{C}^{n}, n \geq 3$.

Since $(f=0)$ is $\omega$-invariant we have that $f \mid(\omega \wedge d f)$ in $\Omega^{2}\left(\mathbb{C}^{n}, 0\right)$, i.e., $\frac{1}{f} \omega \wedge d f$ is holomorphic. Because of the above hypotheses (i) and (ii) we can indeed write $\omega=$ $a d f+f \eta$ for some holomorphic function germ $a$ and holomorphic one-form germ $\eta$. This is the content of the following lemma:

Lemma 4.1. If $X:(f=0)$ is irreducible, reduced and has an isolated singularity (at the origin $0 \in \mathbb{C}^{n}, n \geq 3$ ) then $\omega$ can be written $(*) \omega=a d f+f \eta$ for some holomorphic $a, \eta$.

Proof. This is related to the Dolbeault Cohomology of $\mathbb{C}^{n} \backslash 0$ and the corresponding vanishing theorem of Cartan: $H^{1}\left(\mathbb{C}^{n} \backslash\{0\}, \mathcal{O}\right)=0$ if $n \geq 3$. Locally at any point off the origin we may write $\omega$ as in $\left(^{*}\right)$. This gives an open cover 
$\bigcup_{j \in \mathbb{N}} U_{j}$ of a punctured neighborhood $U^{*}=U \backslash 0$ of $0 \in \mathbb{C}^{n}$. We may assume that $U$ is a polydisc centered at the origin. For each open set $U_{j} \subset U$, the restriction $\left.\omega\right|_{U_{j}}$ writes $\omega=a_{j} d f+f \eta_{j}$ for some holomorphic $a_{j}, \eta_{j}$ in $U_{j}$. We can assume that each intersection $U_{i} \cap U_{j} \neq \varnothing$ is connected. If $U_{i} \cap U_{j} \neq \varnothing$ then on $U_{i} \cap U_{j}$ we have $a_{i} d f+f \eta_{i}=a_{j}+f \eta_{j}$. Thus we have $\left(a_{i}-a_{j}\right) d f=f\left(\eta_{j}-\eta_{i}\right)$. So, because $d f$ does not vanish on $U_{i} \cap U_{j} \subset U \backslash 0$ we have $f \mid\left(a_{i}-a_{j}\right)$ in $\mathcal{O}\left(U_{i} \cap U_{j}\right)$. We write then $a_{i}-a_{j}=f h_{i j}$ for some $h_{i j} \in \mathcal{O}\left(U_{i} \cap U_{j}\right)$. The data $\left\{h_{i j}, U_{i}\right\}$ defines an additive cocycle in $U^{*}$, so that by Cartan's theorem this cocycle has a solution ([6] and [8]). This means that there exist $h_{j} \in \mathcal{O}\left(U_{j}\right)$ such that on each non-empty intersection $U_{i} \cap U_{j}$ we have $h_{i j}=h_{i}-h_{j}$. Therefore $a_{i}-f h_{i}=a_{j}-f h_{j}$. Thus we may define $a \in \mathcal{O}\left(U^{*}\right)$ by $\left.a\right|_{U_{j}}=a_{j}-f h_{j}$. From the above equations we have that $h_{i} d f+\eta_{i}=h_{j} d f+\eta_{j}$. We then define $\eta$ in $U^{*}$ by setting $\left.\eta\right|_{U_{j}}=h_{j} d f+\eta_{j}$. Then on each $U_{j}$ we have $\omega=a_{j} d f+f \eta_{j}=a d f+\eta$. By classical Hartogs' extension theorem ([6] and [7]) the function $a$ and the form $\eta$ extend to $U$ and we write $\omega=a d f+f \eta$ in $U$.

Remark 4.2. The above lemma may also follow from the following argumentation, based on Saito-De Rham division lemma ([16]): From the invariance of $(f=0)$, where $f$ is reduced, we have $\omega \wedge d f=f \theta$ for some holomorphic $\theta \in \Omega^{2}\left(\mathbb{C}^{n}, 0\right)$. This means that $\omega \wedge d f=0$ in the quotient ring $\mathcal{O}_{n} /(f)$. Then, because $\operatorname{sing}(f)=\{0\} \subset \mathbb{C}^{n}$ and $n \geq 3$, we have from [16] (page 166) that $\omega=a d f$ in $\mathcal{O}_{n} /(f)$. In other words, $\omega=a d f+f \eta$ for some holomorphic $\eta \in \Omega^{1}\left(\mathbb{C}^{n}, 0\right)$.

Given $\omega=a d f+f \eta$ we investigate under which conditions $a$ is a unit.

Claim 4.3. Assume that $\omega=a d f+f \eta$ and that:

(Is.3) the algebraic multiplicities of $\omega$ and $f$ at the origin satisfy $\nu(\omega)=\nu(d f)$.

Then the function $a$ is a unit.

Proof. We have $\omega=a d f+f \eta$. From this equation we conclude that $a$ is a unit, simply by comparing the orders of $f$ and $d f$ at the origin, plus using the fact that $\omega$ and $d f$ have the same order at the origin.

From now on we assume that $\omega=a d f+f \eta$ where the function $a$ is a unit. Next we show that $\operatorname{sing}(\omega)=\{0\}$. We can suppose that $a=1$; note that $f$ is a submersion outside $\{0\}$. This implies that $\operatorname{sing}(\omega) \cap f^{-1}(0) \subset\{0\}$. Suppose that $\operatorname{sing}(\omega)$ contains a curve parametrized by $t \mapsto \gamma(t), t \in(\mathbb{C}, 0)$. Then $f \circ \gamma(t) \not \equiv 0$ and up to reparametrization we can suppose that $f(\gamma(t))=t^{p}$ for some $0<p \in \mathbb{N}$. Then $0=\gamma^{*}(\omega)=p t^{p-1} d t+t^{p} \gamma^{*}(\eta)$. This implies $0=p d t+t \gamma^{*}(\eta)$, a contradiction.

Remark 4.4. Now we examine another condition:

(Is.3') For a generic plane section $E:\left(\mathbb{C}^{2}, 0\right) \hookrightarrow\left(\mathbb{C}^{n}, 0\right)$, the restriction $E^{*}(\omega) \in$ $\Omega^{1}\left(\mathbb{C}^{2}, 0\right)$ defines a foliation which is non-dicritical with a singularity of generalized curve type $([2])$ at the origin $0 \in \mathbb{E}^{2}$. 
Then, from [2] we have that (Is.3') $\Longrightarrow$ (Is.3). Indeed, it is possible to give some further conditions on $\mathcal{F}(\omega)$ in order to conclude that conditions (Is.3) and (Is.3') are equivalent (see the paragraph preceding Theorem 1.6 below).

\subsection{Conclusions}

Let us collect our conclusions from the previous discussion:

\subsubsection{Isolated singularity}

We know from the above discussion that for the case of an irreducible hypersurface $X:(f=0)$ at $\left(\mathbb{C}^{n}, 0\right), n \geq 3$; with an isolated singularity at the origin, there always is a holomorphic first integral provided that some condition on the coefficients is satisfied. Indeed:

Proposition 4.5. Let $X:(f=0) \subset\left(\mathbb{C}^{n}, 0\right), n \geq 3$ be irreducible with an isolated singularity at the origin. Given a holomorphic integrable one-form $\omega \in \Omega^{1}\left(\mathbb{C}^{n}, 0\right)$ with $\operatorname{cod} \operatorname{sing}(\omega) \geq 2$ leaving $X_{f}$ invariant, we can write $\omega=a d f+f \eta$. If a is a unity then we have $\operatorname{sing}(\omega) \subset\{0\}$ and we have a germ of a non-constant holomorphic first integral for $\omega$.

As an application, let be given a holomorphic integrable germ of a one-form $\omega=a d f+f \eta$ with $a \in \mathcal{O}_{n}$, for instance if $X_{f}$ has an isolated singularity at the origin (Lemma 4.1). Assume that $X_{f}$ is the only invariant hypersurface and that for a generic plane section $E$ : $\left(\mathbb{C}^{2}, 0\right) \hookrightarrow\left(\mathbb{C}^{n}, 0\right)$, the induced foliation $E^{*} \mathcal{F}(\omega)$ is a nondicritical generalized curve in the sense of [2]. Then the algebraic multiplicities of $\omega$ and $f$ at the origin satisfy $\nu(\omega)=\nu(d f)$ (cf. [2]). Therefore $a$ is a unit (Claim 4.3). From the above, $\operatorname{sing}(\omega)=\{0\}$. By Malgrange's theorem (for $n \geq 3$ ) we conclude that $\mathcal{F}(\omega)$ admits a holomorphic first integral. In short we have:

Proposition 4.6. Given a holomorphic integrable one-form $\omega$ at $0 \in \mathbb{C}^{n}, n \geq 3$, assume that:

(1) $X_{f}:(f=0)$ is irreducible and invariant, where $f$ has an isolated singularity at the origin.

(2) The algebraic multiplicities of $\omega$ and $f$ at the origin satisfy $\nu(\omega)=\nu(d f)$, for instance, if $X_{f}$ is the only invariant hypersurface and for a generic plane section $E:\left(\mathbb{C}^{2}, 0\right) \hookrightarrow\left(\mathbb{C}^{n}, 0\right)$, the induced foliation $E^{*} \mathcal{F}(\omega)$ is a non-dicritical generalized curve.

Then there exists a germ of a holomorphic first integral for $\omega$. 
Remark 4.7. In dimension $n \geq 3$ the fact that $f$ has an isolated singularity at the origin already implies that $X_{f}$ is irreducible. Condition (2) above is satisfied if $X_{f}$ is the only invariant hypersurface and if generic plane sections of $\mathcal{F}(\omega)$ are non-dicritical generalized curves.

Proof of Theorem 1.6. In view of Proposition 4.6 (see also Remark 4.4) it remains to show the "only if" part. Assume that $\omega$ has a holomorphic first integral and $(f=0)$ is the only invariant hypersurface. Let us prove that $\nu(\omega)=\nu(d f)$. Indeed, if $F$ is a holomorphic first integral for $\omega$ then we can assume that $F=g . f$ for some holomorphic $g$. Since $F$ is a first integral and $\operatorname{cod} \operatorname{sing}(d F) \geq 2$, we can write $([16]) \omega=b . d F$ for some unit $b$. Taking derivatives we obtain $\omega=b . g d f+f b . d g$. Notice that b.g is also a unit. Therefore, we must have $\nu(\omega)=\nu(d f)$.

\section{Some relative cohomology}

Let us give now in details the proof of Lemma 2.2. We consider $f \in \mathcal{O}_{n}, n \geq 3$ with $f(0)=0$ and put $X_{f}:(f=0) \subset\left(\mathbb{C}^{n}, 0\right)$. We consider $\omega \in \Omega^{1}\left(\mathbb{C}^{n}, 0\right)$ an integrable germ of holomorphic one-form. We assume that $X_{f}$ is irreducible.

Lemma 5.1. Assume that $\operatorname{cod} \operatorname{sing}(d f) \geq 2$. The following conditions are equivalent:

(1) $d \omega \wedge d f=0$.

(2) $\omega$ is closed on each fiber of $f$.

Proof. It is sufficient to prove the lemma at a generic point (for ad-hoc representatives of our germs). By Poincaré lemma, Lemma 5.1 is true for a submersion. In particular, it is true at a generic point for $f$.

We recall that if $f$ is reduced then $\operatorname{cod} \operatorname{sing}(d f) \geq 2$.

Proposition 5.2. Assume that $\operatorname{cod} \operatorname{sing}(f) \geq 2$. Then the following conditions are equivalent for $\omega \in \Omega^{1}\left(\mathbb{C}^{n}, 0\right)$ :

(i) $d \omega \wedge d f=0$ and $\oint_{\gamma} \omega=0$ for each cycle $\gamma$ contained in a non-singular fiber $f^{-1}(c), c \neq 0$.

(ii) $\omega=a d f+d h$ for some $a, h \in \mathcal{O}_{n}$.

Proof. Since the sense (ii) $\Longrightarrow$ (i) is trivial, we shall assume that $d \omega \wedge d f=0$ and $\oint_{\gamma} \omega=0$ for all cycle $\gamma$ contained in the non-singular fibers of $f$. By the preceding lemma we have that the restriction of $\omega$ to these fibers is closed.

Claim 5.3. There exist holomorphic functions $a, h: \mathbb{C}^{n} \backslash X_{f}, 0 \rightarrow \mathbb{C}$ such that $\omega=a d f+d h$. 
Proof. We will follow an integration argument like in the proof of Theorem II in [1] (see pages 405, 406 and 412-414). Nevertheless, because of the extension problem to the hypersurface $X_{f}$, let us give details about the construction of the function $h$.

We take any point $p \in X_{f}^{*}=f^{-1}(0) \backslash \operatorname{sing}\left(X_{f}\right)$ and a small disk $\Sigma$, centered at $p$, transverse to $X_{f}$ (and therefore to $\omega$ ).

Because $X_{f}$ is irreducible, $X_{f} \backslash \operatorname{sing}(f)$ is connected and we conclude that, for $\Sigma$ small enough, the union $\bigcup_{z \in \Sigma \backslash\{p\}} f^{-1}(f(z))$ is a neighborhood of the origin minus $X_{f}$, and $\bigcup_{z \in \Sigma} f^{-1}(f(z))$ is a neighborhood of the origin. Now we start by defining $h$ in $\Sigma$ as $h(p)=0$ and $h(z)=f(z), \forall z \in \Sigma$. We then extend $h$ to each fiber $f^{-1}(f(z)), z \neq p$ by integration, i.e.,

$$
h(w)=h(z)+\left.\int_{z}^{w} \omega\right|_{f^{-1}(f(z))}=f(z)+\left.\int_{z}^{w} \omega\right|_{f^{-1}(f(z))}, \quad \forall w \in f^{-1}(f(z)) .
$$

This line integral is well-defined due to the condition $\oint_{\gamma} \omega=0$ in (i) in the statement. Thus we have defined $h$ in $U \backslash X_{f}$ for some neighborhood $U$ of $X_{f}$ in $\mathbb{C}^{n}, 0$.

Notice that by definition we have $h$ holomorphic in the fibers $f^{-1}(z), z \neq 0$ and in the transverse disc $\Sigma$. So it is not difficult to conclude that (by a theorem of Hartogs) the function $h$ is holomorphic in $U \backslash X_{f}$. Now we observe that by construction $d h$ and $\omega$ coincide along the fibers $f^{-1}(z), z \neq 0$. Therefore we can write $\omega=d h+a d f$ for some holomorphic function $a: U \backslash X_{f} \rightarrow \mathbb{C}$ (notice that $d f$ is nonsingular in $\left.U \backslash X_{f}\right)$. Since $\omega \wedge d f=d h \wedge d f$ and $X_{f}$ is $\omega$-invariant, we conclude that $(d h \wedge d f)(z) \rightarrow 0$ as $z \rightarrow X_{f}$. In other words $h(z) \rightarrow 0=h(p)$ as $z \rightarrow X_{f}$. In particular $h: U \backslash X_{f} \rightarrow \mathbb{C}$ is bounded and by Riemann extension theorem $h$ admits an unique holomorphic extension to $X_{f}$. This extension satisfies $h\left(X_{f}\right)=\{0\}$.

Once we have $\omega=a d f+d h$ with $\omega$ and $f, h$ holomorphic in $U$, the same holds for $a$ because $a d f=\omega-d h$.

This completes the proof of Proposition 5.2.

Proof of Lemma 2.2. Lemma 2.2 is now a straightforward consequence of Proposition 5.2 and Lê-Saito Theorem (Theorem 2.1).

\section{References}

1. Berthier, M. and Cerveau, D., Quelques calculs de cohomologie relative, Ann. Sci. Éc. Norm. Supér. 26 (1993), 403-424.

2. Camacho, C., Lins Neto, A. and Sad, P., Topological invariants and equidesingularization for holomorphic vector fields, J. Differential Geom. 20 (1984), $143-174$. 
3. Cerveau, D. and Mattei, J.-F., Formes Intégrables Holomorphes Singulières, Astérisque 97, 1982. MR 86f:58006.

4. Cervenu, D. and Moussu, R., Groupes d'automorphismes de $(\mathbb{C}, 0)$ et équations différentielles $y d y+\ldots=0$, Bull. Soc. Math. France 116 (1988), 459-488.

5. Deligne, P., Le groupe fundamental du complément d'une courbe plane n'ayant que des points doubles ordinaires est abélien, in Séminaire Bourbaki 79/90, nov. 79 .

6. Gunning, R. C., Introduction to Holomorphic Functions of Several Variables, Vol. I, Function Theory, Wadsworth \& Brooks/Cole Advanced Books \& Software, Pacific Grove, CA, 1990.

7. Gunning, R. C., Introduction to Holomorphic Functions of Several Variables, Vol. II, Local Theory, Wadsworth \& Brooks/Cole Advanced Books \& Software, Monterey, CA, 1990.

8. Gunning, R. and Rossi, H., Analytic Functions of Several Complex Variables, Prentice Hall, Englewood Cliffs, NJ, 1965.

9. Kaup, L. and Kaup, B., Holomorphic Functions of Several Variables: An Introduction to the Fundamental Theory, De Gruyter Studies in Mathematics 3, in coop. with Barthel, G., 1983.

10. Dung Tráng, L. and Saito, K., The local $\pi_{1}$ of the complement of a hypersurface with normal crossings in codimension 1 is abelian, Ark. Mat. 22 (1984), 1-24. MR 735874 (36a:32019).

11. Loray, F. and Meziani, R., Classification de certains feuilletages associés à un cusp, Bol. Soc. Bras. Mat. [Bull. Braz. Math. Soc.] 25 (March 1994), 93-106.

12. Malgrange, B., Frobenius avec singularités, 1. Codimension un, Publ. Math. Inst. Hautes Études Sci. 46 (1976), 163-173.

13. Malgrange, B., Frobenius avec singularites. 2. Le cas general, Invent. Math. 39 (1977), 67-90.

14. Mattei, J. F. and Moussu, R., Holonomie et intégrales premières, Ann. Sci. Éc. Norm. Supér. (4) 13 (1980), 469-523.

15. Milnor, J., Singular Points of Complex Hypersurfaces, Ann. Math. Studies 61, Princeton Univ. Press, 1968.

16. Saito, K., On a generalization of de Rham lemma, Ann. Inst. Fourier 26 (1976), 165-170.

Dominique Cerveau

Université de Rennes I - IRMAR

Campus de Beaulieu

FR-35042 Rennes Cedex

France

dominique.cerveau@univ-rennes1.fr

\author{
Bruno Scárdua \\ Instituto de Matemática \\ Universidade Federal do Rio de Janeiro \\ Caixa Postal 68530 \\ CEP. 21945-970 \\ Rio de Janeiro, RJ \\ Brasil \\ scardua@im.ufrj.br
}

